\title{
Abenteuer eines Alchemisten aus dem 17. Jahrhundert.
}

\author{
Von E. FINK.
}

Unter den Bestallungsakten aus der Zeit des Osnabrücker Bischofes Ernst August I. (1662-1698) befindet sich ein undatiertes Schriftstück, ${ }^{1}$ ) das auf einem losen Umschlag die von einer Kanzleihand geschriebene Aufschrift "Martin Apollo Dantzigs Gesuch betr." trägt. Innen liegen 27 beschriebene Folioseiten, auf denen aber nur die Unterschrift des Bewerbers eigenhändig zu sein scheint. Am unteren Rande der ersten Seite steht der kurze Aktenvermerk: "Ist ohne resolution hinzulegen befohlen worden", auf der Rückseite des Umschlagblattes die Aufschrift Species facti. Es ist also nicht das Bewerbungsgesuch selbst, das uns vorliegt, sondern nur eine Anlage $\mathrm{zu}$ ihm, durch welche der Einsender offenbar seine Qualifikation für die offene Stelle zu erhärten sucht. Dantzigs Heimat ist Clausthal im Harz, wo sein verstorbener Vater Stadtschreiber gewesen ist. Die geringe Aussicht auf die Nachfolge in diesem Amte heißt ihn nach Halle gehen, um dort Medizin zu studieren. Hier fällt er einem Charlatan in die Hānde, dem Schreiber Frenckel, der ihn in die Geheimnisse der Chemie oder Alchemie, wie man damals zu sagen pflegte, mit Erfolg einweihte und ihm schließlich verspricht, gegen einen baren VorschuB von 1000 Talern angemessene Beschäftigung in der mitgeteilten Wissenschaft bei vornehmen Herren zu verschaffen. Beide halten sich zu diesem Zwecke vorübergehend an zwei schlesischen Adelshöfen auf und begeben sich von dort

1) Original im Kgl. Staatsarchiv zu Osnabrïek, Landesarchiv Bestallungsakten Lit. D. 
über Dresden nach Arnstadt, wo dem M. A. Dantzig eine Stelle als Bergrat neben sonstigen gewinnversprechenden Aussichten aus den alchemistischen Studien winkt. Trotz alledem scheint ihm das Leben in der kleinen thüringischen Residenz nicht behagt zu haben, und die Möglichkeit, die bescheidenen Verhältnisse in Arnstadt mit den vorteilhafteren Lebensbedingungen an einem angesehenen Bischofssitze vertauschen zu können, scheint ihn zu einer Bewerbung nach Osnabrück veranlaßt zu haben.

Die Anlage dieses Gesuches gibt nun als Species facti eine Schilderung der etwas abenteuerlichen Schicksale, die der thüringische Bergrat angeblich seit seiner Ausreise von Halle bis zur Anstellung in Arnstadt erlebt haben will. Er stellt sich als viel begehrte Persönlichkeit hin und spielt sich als Opfer eines Intriguenspieles auf, das sein Kumpan, der Schreiber Frenckel, aus gewinnsüchtigen Absichten wider ihn in Dresden angezettelt habe; daneben vergißt er nicht, an den geeigneten Stellen seines Berichtes Proben seiner alchemistischen Kenntnisse zu geben. Nichts destoweniger haben die Geheimen Räte in Osnabrück, wie der Kanzleivermerk "Ist ohne resolution hinzulegen befohlen worden" verrät, sein Gesuch ohne weiteres zu den Akten legen lassen. Für derartige zweifelhafte Existenzen vom Schlage des Bewerbers hatte man hier zweifelsohne wenig Verständnis. Selbst die Ankündigung, das aurum potabile herstellen zu können, jenes Universalmittel, welches nach damaliger Anschauung in angemessener Verdünnung als Trinkgold alle möglichen Leiden $\mathrm{zu}$ heilen imstande war, sogar das Leben verjüngte und verlängerte, ebenso die viel versprechende Zusicherung, Gold und Silber aus unedlen Metallen gewinnen zu können, haben der Bewerbung keine empfehlende Wirkung beizulegen vermocht. Ist es Zufall, daß die beiden Freunde immer gerade dann ihre Stelle wechseln, wenn einer von ihnen seine gepriesene Kunst zeigen soll, nachdem er zuvor Reisevorschüsse und sonstige Geldbeihilfen $z u$ erbetteln verstanden hat? Die Hoffnung, in Osnabrück in gleicher Weise wirken zu können, erfüllte sich hier nicht.

Von kulturhistorischem Interesse ist diese Species facti dadurch, daß sie uns zeigt, in welcher Weise einerseits gerade die ersten Kreise der Gesellschaft von der Sucht der Gold- 
gewinnung ergriffen waren, und anderseits, in welcher Weise die Jünger dieser vermeintlichen Kunst diese Leidenschaft und Kurzsichtigkeit ihrer Opfer auszubeuten verstanden. Ist es auch nichts Neues, was wir aus dem nachfolgenden Bericht ersehen, so dürfte seine Wiedergabe schon deswegen lohnend sein, weil er als ein unmittelbares Zeugnis aus der Feder eines Alchemisten einige interessante Streiflichter auf das Leben und Treiben der Goldmacher wirft.

Nach meines Seel. Vatters tode, gewesenen Stadtschreiber zum Claußthal, alB zwischen dem Hochwollgebohrnen Herrn Berg Haubt Mann von dem Busch und Richter und Raht daselbst de jure vocandi et confirmandi eines neuen Stadtschreibers gestritten wurde, fand ich unter andern Brieffschaften einige, woraus klar zu sehen, daß das jus confirmandi jeder Zeit bey denen Herrn Berg Hauptleuthen bestanden. Diese brieffe übergab ich an den Herrn Berg-Hauptmann von dem Busch in Meinung, es würde mir diese vacante Bedienung aufgetragen werden, weiln ich von den Seel. Vatter fast $8 \mathrm{Jahr}$ hindurch in Verwaltung dieser Bedienung bin unterrichtet worden, allein ich hatte mir dadurch Richter und Raht zu Feinde gemacht; dahero verschwand mir alle Hoffnung zur zukünftigen Beförderung, resolvirte mich dieser wegen, nach Halle zu reisen, umb daselbst mich in studio Medico unterrichten zu laBen. Daselbst werde ich bekand mit dem FloB-Schreiber Christian Frenckel, welchen ich recommendiret worden. Dieser giebet mir zu verstehen, weil er vernommen, daß ich ein liebhaber der Probier Kunst, undt weiln in diesem studio unter der Hand viel Curiosa, welche das Feuer offenbahret, erscheinen und die untersuchung dergleichen Sachen keines Privat Person Werck ist, weiln es Zeit, Gelegenheit und Geld erfordert - daß wann ich ihme, do ich durch den Seegen Gottes etwas profitables in studio Chymico solte ausrichten, aus seiner Noht erretten wolte, welche sich etwann auf tausend Thlr. belauffen möchte, so wolte er mich zu einem raisonnablen liebhaber dieser Kunst alB zur Ihr. Hochgräffl. Gnaden von Hoditz nacher Schlesien mitnehmen, bey welchen Graffen er schon einmahl gewesen, hette auch demselben versprechen müßen, eine persohn, welche das Probieren verstünde, 
zu recommendiren, weil der Herr Graff verschiedene Bergwercke beseBe, welche nicht im Stande. Ich ließ mir diesen Vorschlag gefallen, weil ich ohne dem meine fortun suchte, reisete mit Frenckeln nach obgedachten Graffen, beschloß bey mir, wann ich etwa daselbst meine employe nicht finden solte, nach Ungern zu gehen und daselbst die Bergwercke und deren Tractament zu untersuchen. Wir reisen beyde zu Ihro Hochgräffliche Gnaden nach Roßwalde, wurden nach unserer Ankunft zur Taffel gebethen; meine wenige Person stund den Herrn Graffen an, Sie wolten mich zu dero secretario annehmen. Ich solte mich aber ad dies vitae $\mathrm{zu}$ beständigen Diensten obligiren und wolten mir jährlich 100 Schlesier-Thlr. nebst freyer Taffel bey Ihro [Gnaden] geniesen laßen, dabey wolten wir allerhand Curiosa in Chymicis versuchen, und wann durch den Seegen Gottes einiger profit herauskäme, solte ich von allen Arbeiten tertiam partem zu geniesen haben. Diese gethane Vorschläge hette ich vor meine Persohn auf eine gewiße Zeit acceptiret; weiln aber Frenckel anfing zu quaeruliren, daß ihme dadurch nicht gleich geholffen were, sprach er den Graffen an, daß man ihme mögte mit 1000 Thlr, an die Hand gehen; er wolte sein Hauß und Garten zur Hypothec verschreiben. Allein der Herr Graff schlug ihme solches schlechterdings ab. Dieses verdro $\beta$ den Frenckel sehr, persuadirte mich, die vorgeschlagene conditiones des $\mathrm{Hh}$. Graffen zu refusiren, mit versprechen, daß er mir wolte beßere recommendation machen bey einem Baron nahmens Schmeroffski. Auf Frenckels vielfältiges anhalten resolvirte endlich, mit demselben zu gedachten Baron zu reisen, wurden auch nach unser Ankunfft zur Taffel gebethen.

Bey diesem Baron hielt sich ein Haubt Mann auf nahmens Herr von Vorbeck. Dieser Haubtmann hatte sowoll von vorgedachten Herrn Graffen alB auch diesem Baron viel 1000 Thlr. genoßen vor zwey Chymische Proceße. Nach der Taffel ward ich von dem Baron undt dem Haubtmann in das Laboratorium geführet. Daselbst wurde ich gewahr, daß der Haubtmann dem Spiritum Mercurii ${ }^{1}$ ) aus der Ungrischen Minera Vitrioli nicht könte zu wege bringen, weiln diese Minera nicht gereiniget

1) Mercurius $=$ Quecksilber. 
war, fraB also der bey der Minera sich noch befindente Alaun alle Gläßer durch, weswegen auch der Baron sehr ungehalten wurde; trug mir auf, den Haubtman zu hinterbringen, daß es bald Feurabend werden solte. IndeBen bekommt der Haubtmann brieffe, $\mathrm{da} B$ aus Ungern allerhand schöne Mineralien an der Bielitzer Gräntze wären ankommen. Ich ward von ihm ersuchet, mit dahin zu reisen; ich resolvirte mich par Curiosite, dergleichen Ungrische Mineralien zu Gesicht $z \mathfrak{u}$ bekommen, reise mit demselben. AlB wir an obgedachten Orth kamen, fanden sich keine Mineralien. Ich machte mir dahero allerhand wunderliche Gedancken von diesem Haubtmann. Nach zweyen Tagen komt Frenckel nachgereiBet und von dem Baron an mich expediret mit ansuchen, daß ich doch eyligst mochte zuruckkommen undt des Haubtmanns Processe untersuchen, damit er möchte Gewibheit haben, ob die erkauften Processe richtig; ich solte davor ein gratial zu gewartten haben. Ich reisete darauf sofort mit Frenckeln zu dem Baron, wurde mit demselben einig vor die untersuchung der erhandelten Processe umb 80 Thlr., und solte der ProceB zur Tinctur ${ }^{1}$ ) in 3 Theile getheilet werden, davon ich einen partem genieBen solte. Der andere proceB mit der Cementation des Silbers ${ }^{9}$ ) gab die Marck eine quente ${ }^{8}$ ) Luna fixa ${ }^{4}$ ) anstadt des versprochenen Goldes, welches mit einem exaltirten Golde gar leicht zur perfection hette können gebracht werden. Alleine ich wolte solches dem Baron nicht offenbahren. Ich untersuchte indeBen den Proce $B$ de Tinctura aufs fleißigste, fand aber denselben in fundamento nicht gegründet, muste dahero an den Haubt Mann schreiben, daß er sich einfinden und praestanda praestiren solte. Der Haubt Mann verlangte zuvor eine versicherung, daB man ihm nicht arretiren wolle, wann er wieder kommen solte. Der Baron committirte mir abermahlen, den Haubtmann zu antwortten, doferne er in seinem GewiBen richtig, dürfte er sich nichts besorgen. Alleine der Haubtmann wolt sich nicht wieder einfinden.

1) $\mathrm{Ob}$ unter Tinctura vielleicht die Feststellung desjenigen Mittels zu verstehen ist, durch welches aus unedlen Metallen Oold oder Silber gewonnen werden konnte? Vgl Schmieder, Oesch. der Alchemie, Halle 1832, Seite 2.

ク) Zementation des Silbers = Reinigung des Silbers durch Glühen mit Zement.

9) Eine Quente $=1$ Drachme $=3,75 \mathrm{gr}$.

t) Luna fixa $=$ reines Silber. 
E. Fink.

Unterdeßen nahmen wir einen Proceß vor aus dem 이 $^{1}$ ) umb mich so lang bey dieser Gelegenheit $z \mathfrak{u}$ souteniren, bis Frenckel, welchen ich nacher Arnstadt geschicket, wieder revertirte, umb daselbst bey dem Cammerdiener Edellmann zu vernehmen, ob er meinentwegen mit seinem Fürsten gesprochen und, wie wir abgeredet, sein Wortt gehalten. Frenckel hat die Gnade, mit Ihr. Durchl. zu Arnstadt selbst zu sprechen, worauf Ihro Durchl. dem Cammerdiener Edellmann befehlen, daß er diesen abgeschickten Frenckel geschwinde mit einen Schreiben nebst übersendung der reisekosten an mich wieder expediren solle, deßen inhalt, ich solle mich geschwind zur reiße begeben und eine gewiße Bedienung antreten. Diese erfreuliche Zeitung und Ankunft des Frenckels gab mir Uhrsach, dem Baron Schmeroffski mein bevorstehendes Glück zu entdecken, wolte den angefangenen Kupfer ProceB nicht ferner elaboriren. Der Baron wolte mich nicht reisen laßen, gab vor, daB er mich an Ihr Kayserl. Majestät recommendiren wolle, alwo es mir viel beßer glücken solte, weiln die andern Fürsten alle von Ihro Kayserl. Majestät dependirten, und was dergleichen persuasiones mehr waren. Ich wäre auch woll geblieben, wenn ich wegen der Religion nicht so sehr wäre verkätzert worden, daß man auch allen Versuch thäte, mich zur Catholischen Religion zu bringen. Alleine ich resolvirte mich kurtz, ließ mir in Troppau nebst den Frenckel einen $\mathrm{Pa} \beta$ geben, und weiln damahls wegen der Pest bey Breslau die paßagiers nicht woll fortkommen konten, reiseten wir an den Bömischen Gräntzen über Greiffenberg in unser Vaterland.

Wir kamen zu Bautzen an, ließen uns daselbst neue Päße geben und war ich willens, über den langen Hohn nacher Leipzig und Arnstadt zu reisen. Frenckel persuadiret mich listig, mit ihm auf Dresden zu reisen. Ich reise mit denselben auf Dresden zu. AlB wir nun vor der vorstadt kamen, musten wir unsere päße vorzeigen, welche vor richtig erkand; bekamen darauf ein jeder einen Passirzettel. AlB wir aber vor das Stadt Thor kamen, wurden unsere Päße durch einen Soldaten hinein getragen. Ich muste fast 2 Stunden vor dem Thore wartten. Endlich kam der Thorschreiber, fragte, wie ich hieße. Kurtz darauf wurde ich zu

1) Vgl. Seite 62 Note 4. 
dem Pest Commissario gefodert. Dieser fragte, wo der alte $\mathrm{Pa} \beta$. were. Ich remonstrirte darauf, wie daß ich nebst den Frenckel und noch einem Mann einen Paß zusammen genommen, in Bautzen aber, weil wir willens waren, von einander zu gehen, ließ sich ein jeder seinen eigenen $\mathrm{Pa}$ geben. $\mathrm{AlB}$ ich nun befraget wurde, wo der alte $\mathrm{PaB}$ were, wuste ich nicht, ob ihn Frenckel zerrißen oder ob er ihn zu Bautzen gelaßen. Der Commissarius sagt hierauf, daß weiln ich zweyerley reden geführet, könte er mich nicht paBiren laßen. Ich remonstrirte darauf, warum man dann den Frenckel und den andern Mann, welche mit mir aus Schlesien gereiset, auf ihren $\mathrm{Pa} B$ paßiren ließ, da wir drey auf einen $\mathrm{Pa} \beta$ zusammen gestanden, in Bautzen aber hat sich ein jeder seinen eigenen $\mathrm{Pa} \beta$ geben laßen, mein $\mathrm{Pa} \beta$ were ebenso richtig alß der andern ihrer. Allein der Pest Commissarius antworttet mir: ihr höret wol, ihr solt nicht paßiren. Ich ging indeßen vor das Tohr ins wirthshauß. Frenckel aber und der andere Mann wurden paBiret und, so viel ich vernommen, hat derselbe mit Dr. Glasern, den Cammer Fiscal, unterredung gepflogen. Nach einer halben Stunde kombt Frenckel, hohlet mich aus dem Wirthshaub, vorgebend, es sey resolviret, daß ich solle paBiren, worauf wir uns in der Stadt in den Schwan einlogirten. Es fehlete mir an eintzein Gelde, lieb dieser wegen durch dem Mann, welcher mit uns aus Schlesien gereiset, ein loht Gold verkauffen, welches ich extraordinair durch das Antimonium erhöhet hatte. Der Gold Schmid, der solches gekauffet, saget, man möchte ihm dergleichen Gold mehr bringen, er hette lebenslang dergleichen nicht unter Händen gehabt, gehet sofort nach der Obrigkeit und zeiget solches an, worauf der Mann vorgefodert und scharff examiniret wird, ob dieses verkaufte Gold nicht durch Kunst gemachtes Gold were? Ille saget nein; es were so hoch und fein, weiln es durch das Antimonium gegoßen were. Indeßen komt Dr. Glaßer der Cammerfiscal nebst seinem Sohne in das WirthshauB; en regard des Frenckels, weil diese mit einander gevattern waren, redete ich mit ihnen. Unter andern discursen bringet Doctor Glaser vor, wie Er von Frenckel vernommen, daß ich ein liebhaber der Chymie were. Er hette sein Hauß, welches er in Leipzig gehabt, drauf gewandt, schätzte solches woll auf 14000 Thir. und 
E. Fink.

hette noch keine müglichkeit befunden. Ich besorgte mir von diesem Manne nichts böses, zeigete ihme einer Erbsen groß Gold, welches aus dem Schweffel des Silbers hervor gebracht ware, dabey remonstrirte, wie wunderbahr diese Kunst, daß der Schweffel des Silbers könne ander Silber in Gold tingiren, ob schon kein profit dabey were. Jedennoch sey es eine wunderns würdige Sache und steckte dahinter viel Geheimniß verborgen. Ich zeigte auch demselben 1 quente der lunae fixae, welches der Ambtmann in Dresden noch behalten.

Nachdem ich nun eine weile mit ihme von allerhand Curiosen dingen gesprochen, gingen Sie wieder von mir; besorgte mir dieserwegen nichts. Alleine kurtz darauf komt der Commissarius mit sich bringend einen granadier, welcher sich vor der Hauß Thür niedersetzen muste. Dieser Commissarius fodert von mir den Pas und PaBir Zettel zurück, mit vorgeben, daß die Geheimbten Rähte den $P a ß$ noch einmahl zu sehen begehrten, befohl den granadier, ein wachsames Auge auf mich zu haben. Gegen abend kam der Commissarius wieder, redete mit dem Wirth heimblich und nahm den Granadierer wieder mit sich. Des andern Tages frühe komt der Commissarius abermahl nebst zweyen granadierer, saget mir, daß weiln ich zweyerley reden geführet von wegen des Paßes, also were der Geheimbten Rähte Befehl, daß ich solte so lang arretiret werden, bis ein attestat von dem Raht zu Bautzen mir procurirte, daB der $\mathrm{PaB}$ richtig, welchen ich von Bautzen mitgebracht; befohl dabey, mit keinen Menschen ein Wortt zu sprechen, befohl auch denen beyden granadierern, dahin zu sehen, daß ich mit niemanden $z u$ sprechen käme.

Ich war indeßen besorget umb ein Attestat von Raht zu Bautzen, schickte den Frenckel dahin, welcher auch ein Attestat bekommen, $\mathrm{daB}$ ich mit richtigen $\mathrm{PaB}$ were von Greiffenberg ankommen. Indeßen kombt der Commissarius alle tage, forschet fleißig, ob ich derer Geheimbten Rähte befehl nachlebe. Es begab sich, daß einsmahls einige Gäste in diesem Gasthoffe versamlet waren, redet mich einer von ihnen an, so daB ich ihm nicht umbhin konte unbeantworttet [zu] laßen. Indem nun solches geschieht, eröffnet der Commissarius die Thür, siehet, daß ich rede. Dieser 
fährt auf mich mit wortten, $\mathrm{Er}$ sehe woll, daß ich der Geheimbten Rähte befehl nicht respectirte, man wolte mich anderst wohin bringen. Ich hoffe aber mit schmertzen auf des Frenckels Ankunfft von Bautzen, in hoffnung, daß ich nach erhaltenen Attestat wieder solte befreyet werden. Alleine was geschieht? Doctor Glaser nebst seinem Sohne kamen zu mir und besuchten mich. Der junge Glaser schlug mir vor, daß er wolte zu Ihr. Durchl. dem Fürst von Fürstenberg gehen, vor mich daselbst sollicitiren, daB ich loB käme, alleine ich müste ihme und Ihr. Durchl. einige WiBenschaften entdecken. Ich wuste mich zu nichts zu resolviren, sondern beklagte mich, daß es ziemblich würde ins Geld lauffen, wann ich lange solte arretiret werden. Dieses mag der Glaser muhtmaßlich hinterbracht haben. Demselben abend al $\beta$ den dritten Tag meines Arrestes kam der Commissarius mit sich bringend den Amts Actuarium nebst dem Stock Meister ${ }^{1}$ ) und brachten mich, nachdem ich die Wachte bezahlet, in Stockhauß auf der Gehorsam Stube. Ich, der schon vor schrecken halb tod war, muste mit mehrem erfahren, daB der Stock Meister über mir herfuhr, das Kleid aufriß, Taschen, Hosen und Schue, ja alles durch visitirte, alles weg nahm und solches ins Ambt liefferte. Ich muste die harte banck drücken, wuste vor bekummernis und Schrecken nichts anzufangen. Ich hatte einige schöne Collectanea Chymica, welche so umbsonst hingingen, und alß ich dieselbe nach meinem Arrest wieder haben wolte, muste ich erst 5 Thlr. ins Ambt lieffern.

Ich wurde nach zweyen Tagen ins Ambt gefodert und von dem Ambtmann befraget, wo ich herkäme; responsum: aus Schlesien von einem Baron, Schmeroffski genand. Porro quaesitus, was ich daselbst gemacht. Ich erzehlte darauf, daß ich von vorgedachten Baron were ersuchet worden, einige Chymische Proceße zu untersuchen, welche $\mathrm{Er}$ von einem Haubtmann nahmens von Vorbeck umb ein gewiß stück Geld erhandelt hatte. Porro quaesitus, ob ich nicht einen Haubt Mann kennete, nahmens Weideman, und ob ich mit demselben einige brieffe gewechselt? Ego: Nein, wüste mich nicht zu entsinnen, allein es were ein Haubt Mann da geweßen, welcher sich nandte Herr von Vorbeck;

1) Gefängniswärter. 
an diesen Haubtmann hette ich müßen zweymahl schreiben, von dem Baron dazu bevollmächtigt, daß sich der Haubtmann wieder einfinden möchte und seine Sache ausmachen. Ich wurde ferner gefraget, ob ich diesen Haubtmann von Vorbeck wol kennen wolte, wann ich ihm zu sehen bekäme. Responsum: Ja, ich wolte ihn noch wol kennen, wann ich ihme sehe. Der Ambtmann befiehlt, den Haubtmann Weideman herzubringen. AlB derselbe erschien, redete ich ihm an, wie er der Haubtmann von Vorbeck hierher kảme. Ille beklagte sich, daß man ihn hatte ohnweit Schweinitz arretiret und nach Dresden geführet. Ich wurde auch befraget, warum ich vermeinte arretiret zu seyn. Ich wüste keine Ursach alB daß der Commissarius meinen $\mathrm{PaB}$ alß ungiltig gehalten, weiln ich zweyerley reden geführet.

AlB ich nun 14 Tage geseßen, schicket der Ambtman zu mir, ich solle zu ihme kommen. AlB ich ins Ambt kam, hinterbrachte mir der Ambtmann, daß Ihr. Durchl. der Herr Stadthalter Fürst von Fürstenberg ihme hette anbefohlen, mir zu hinterbringen, daß sich Ihr. Durchl. meiner wolten annehmen und mich ehester Tage wolten loß laßen; ich solte mich nicht so sehr bekümmern und muste eine gute weile bey dem Ambtman verbleiben, umb mich in etwas zu recolligiren, weil ich fast halb Tod war vor Bekümmernis und Schrecken.

Acht Tage hernach wurde ich wieder vorgefodert und wurde mir von dem Ambts Actuario ein Rescript vorgelesen aus der Geheimbten Rahts Stube, dieses inhalts: Was aber anlanget Martin Apollo Dantzig, so findet man denselben in keinem puncte beschweret, kan also derselbe, jedoch gegen juratorische Caution, loß gelaßen werden; worauf ich das Eyd ablegen muste, daß ich nicht wolte vor der Stadt gehen, sondern alles leyden, dulten und bezahlen, was Urthel und Recht mit sich brächte, So wahr etc. Ich wie schon gedacht wahr vor schrecken halb todt, konte keine deliberation dieses Eydes nehmen, sondern danckte Gott, daß ich dadurch des unerträglichen Arrestes loß wurde, wo ich nicht gar hette crepiren wollen in diesen Arrest. Nachdem ich nun das mir vorgeschwatzte Eyd nachgeredet, nahm des Dr. Glasers Sohn mich mit sich nach ihrem Hauße, nachdem ich zuvor pro Rescripto $2^{1 / 2}$ Thlr., item dem Stock Meister 5 Thlr., vor jedes verhör 1 Thlr. 
bezahlt hatte; ohne was mir sonst aufgangen, komt mir woll auf 80 Thlr.

Nachdem ich nun einige Wochen her meine halb erstorbene Glieder und Sinne in etwas recolligiret, schickten Ihr. Durchl. der Fürst von Fürstenberg zu Glasern, ließen durch dero Cammerdiener sagen, daß das bewuste Frauenzimmer zu Ihr. Durchl. kommen solte, weil es Ihr. Durchl. wolten geheimb gehalten wißen vor dero Bedienten. Ich verfügte mich dahin, Ihr. Durchl. befanden sich im bette. Ich aber dancke vor die Gnade, daß ich meines Arrestes were loß gelaßen. Ihr. Durchl. fielen mir in die Rede sagend, Ihr seyd ein ehrlicher Mensch, ich habe aus denen Acten ersehen, daß ihr seyd requiriret worden, einige Chymische Proceße zu untersuchen. Ich habe auch ersehen aus den brieff, welcher noch bey denen Acten befindlich, daß ihr nach Arnstadt seyd geruffen. Weil euch aber das Unglück betroffen, daß ihr alhier seyd in Arrest gerahten, so bleibet bey mir. Was wolt ihr bei den (!) armen Fürsten machen; Er hat kein Geld. Ich habe Geld, ihr könnet nur fodern, da ist charta blanque, setzet selber auf, so viel ihr wollet. Ich antworttete darauf, daß ich von Ihr. Durchl, kein Geld verlangte, inmaßen man in untersuchungen derer Chymischen Wißenschaften nicht alle Zeit glücklich were, weiln es kein Handwerck undt kome schlechterdings von Gott; were fast gleich dem Ackerbau, da der bauer, nachdem er den Acker bereitet, würffe er das Korn hinnein und stellete es übrigens den Seegen Gottes anheim. So were es auch in Chymischen verwandelungen, bestünde eben so wenig in unsere Macht. Ihr. Durchl. sagten hierauf, Sie weren kein liebhaber des Spiels, viel weniger des truncks ergeben, weren auch kein liebhaber der Frauenzimmer noch der Music, sondern ein liebhaber des Studii Chymici. Ich solte bey sie bleiben, Sie hetten lust etwas untersuchen zu laßen, und ob es schon nicht allemahl gerieth, so fünde man doch immer etwas neues. Vielleicht möchte ich eine gute Medicin finden.

Ich solte Ihr. Durchl. erzehlen, was ich woll vor Curiositäten gesehen. Ich erzehle, daß ich an einen ungenandten Orth helffe eine Tinctur ausarbeiten auf diese Aart: wir zogen dem Silber seinem (!) schönen blauen Schweffel aus, so blieb ein 
Schneeweises Corpus zurück, aus diesem zogen wir daß Mercurialische Sal Lunae, ${ }^{1}$ ) hernachmahls imbibirten wir dieses Saltz mit einem oleo Metallico und bekamen eine Tinctur. Dabey wurde mir gesaget, daB es auf viel tausend theile könte Multipliciret werden, welche Multiplication ich aber noch nicht versuchet. Von der Tinctur hette ein lot (lt.) 16 lt. tingiret, item mit dem Mercurialischen Sale Lunae hette ich sehen den Mercurium Currentem ${ }^{2}$ ) in fein Silber tingiren, desgleichen hette ich gelernet ein wahrhafftes aurum Potabile ${ }^{3}$ ) aus dem Golde machen, welches per Retortam getrieben gleich dem schönsten bluth herüber ginge, welche eine kostbahre Medicin. Diese tropffen, wann sie auf einen zubereiteten Silberkalck gegoßen und einige Zeit digeriret, geben, nachdem der Silberkalck reduciret, soviel Gold alB dieser Tropffen am Gewicht ausgetragen. Dahingegen wann diese Tropffen auf kein fixes Metall alk auf $\hat{\delta} q p^{2}{ }^{2}+$ und $\not$ rio $\left.^{4}\right)$ getragen würden, geben sie auch nimmermehr Gold; lege also die gantze kunst daran, daß man derer beyden fixen Metallen Solis et Lunae ${ }^{5}$ ) ihr Mercurialisches Sal lunae extrahirte und zur Tinctur praeparirte. Ihr. Durchl. verlangten zu wiBen, was es ohngefehr kostete, dieses $z \mathfrak{u}$ untersuchen, warneten mich anbey, dieses Zeit meines lebens keinem Menschen zu offenbahren, oder ich würde jeder Zeit in Gefahr stehen, und solte dran gedencken, daß es mir ein fürst gesaget hette.

Ihr. Durchl. resolvirten sich, zu den Proceß von der Tinctur des Mercurialischen Sal Lunae 200 Thlr. herzugeben; es stunde mir aber nicht an, bey diesem fürsten einen Laborantz abzugeben, weil ich so aufgefangen undt übel tractiret worden. Stellete derowegen vor, was den Proceß $\beta$ de Tinctura anbelangte, könte ich nicht gut vor seyn, daß derselbe allezeit geriethe, und müste ich besorgen, woferner ich darinn unglücklich und Ihr. Durchl. hetten einige Unkosten und da ich solche nicht restituiren könte - daß ich einen Schümpff davon träge, welches mir nicht anständig, baht hertzlich, daß man mich mögte nacher Arnstadt reisen laßen

1) Über diesen Terminus technicus konnte ich keine Aufklärung erlangen.

2) Mercurius currens $=$ metallisches Quecksilber.

3) Das als Medizin bekannte Trinkgold.

4) Jene vier Zeichen bezeichnen in der obigen Reihenfolge die Metalle Eisen, Kupfer, Blei, Zinn und Quecksilber.

5) Oold und Silber. 
und daselbst die mir offerirte bedienung anzutreten. Allein ich bekam einen solchen Verweiß, daß ich ja daran nicht dencken solte, und gab mir Dr. Glasers Sohn genug zu verstehen, ich solte mir das aus dem Sinne schlagen, widrigen fals würde man mich wieder hinsetzen. Endlich resolvirten sich Ihro Durchl. von Fürstenberg also zu mir sagend: hört mein lieber Sohn, ich verspiele in einem abend auf einen Chartenblatt 100 Ducaten und habe nichts davor zu gewartten, so kann ich auch schon so viel auf die untersuchung eines solchen wercks anwenden; schreibt indeßen euren Fürsten, sobald daß ihr eures Arrestes endlediget weret, würdet ihr euch einfinden. Unterdeßen könnet ihr es ja versuchen, und so es nicht solte eintreffen, so wil ich euch nicht aufhalten, sondern könnet $\mathrm{zu}$ euren fürsten hinnauf reisen.

Endlich kombt auch Frenckel von Arnstadt wieder zurück, welchen ich hatte daselbst an Ihr. Hochfürstl. Durchl. abgeschicket, umb zu hinterbringen, wie man mit mir verfahren. Ihr. Durchl. schicken mir 30 rthlr. reise Geld und solte ich eyligst überkommen. Es trug sich aber zu, daß mir Frenckel einen Mann recommendirte; dieser Mann soll mit einem Künstler in vertrauligkeit leben. Derselbe soll innerhalb 6 Stunden vermittels einer Kugel den Arsenicum figiren können, da $\beta$ er etliche loht fein Silber aus dem Arsenico bringen könte; das loht Silber käme mit allen unkosten auf $\mathbf{4}$ groschen ohne das $\odot$, so noch darinnen were, und vermeinte Frenckel, daß man diese Kunst ümb ein stück Geld von dem Künstler erlernen könte. Ich trug dieses alB eine große Curiosite dem fürsten vor. Dieselben hatten daran einen Gnädigen gefallen, resolvirten mich dahin zu schicken, ümb zu sehen, ob ich diese Kunst von dem Künstler erlernen könte; ließen mir 50 thir. Reisekosten auszahlen und gaben mir einen $\mathrm{PaB}$, darin ich mich vor dero secretario ausgeben muste, sagten mir, ich möchte dahin sehen, daß ich diesen Künstler bis in Leipzig mit mir führen könte, da wolten Ihr. Durchl. von Fürstenberg hinkommen und mit dem Künstler selbsten sprechen oder, da solches nicht seyn könte, wolten Sie zu erlernung dieser Kunst einen Wechsel übermachen. Ich reise von Dresden weg und weil sich der Künstler an keinen gewißen Orth aufhält, komme ich in erfahrung, daB derselbe in Schwartzburgschen landen 
ohnweit der langen wiese. Ich reise dahin, fand aber den Künstler da nicht und weiln ich bey dieser Gelegenheit auf Arnstadt reisete, um bei Ihr. Durchl. daselbst meine unterthänigste aufwarttung zu machen, erzehlte anbey, wie ich were Arretiret worden und were ich willens, von hier nach dem Hartz $\mathrm{zu}$ reisen, umb meine Eltern ${ }^{1}$ ) zu besuchen; von da würde ich wieder auf Dresden gehen. Ihro Hochfürstl. Durchl. zu Arnstadt schoben mir darauf ins Gewißen, daß weil ich mich zu dero Diensten schon vor einiger Zeit resolvirt hette, wie die brieffe wiesen, auch jeder Zeit bestandige Dienste vor andern gingen, wolten mich Ihr. Durchl. nicht wieder weg laßen, sondern ersuchten mich, dero Berg Werke zu befahren und davon einen Bericht abzustatten, welches ich auch gethan; wurde nechst diesem auch ersuchet, die laboratoria Chymica zu untersuchen, gab davon nach meiner wenigkeit gründliche Nachricht, welches naturgemäß elaboriret würde und welches nur vergebene Unkosten veruhrsachte.

Nachdem solches geschehen, offerirten mir Ihr. Durchl. die Berg-Rahts Charge. Ich habe solche in tantum angenommen mit der Reservation, daß wan ich von Fürst von Fürstenberg nichts zu besorgen, worauf mir Ihr. Durchl. vorstelleten, wann Sie zu Dresden an meine Person was unrechtes zu praetendiren gehabt hetten oder noch gedächten, so könten sie mich dieser wegen zu Arnstadt belangen; zudem wer es eine Sache, welche man per mandatarium könte ausführen laßen. Ich solte mir dieser wegen keine Bekümmernis machen; wann Sie was gegen mir gefunden hetten, sie würden mich nicht $1 / 4$ jahr lang nach meinem Arrest frey haben herumb gehen laßen. Und so auch der Baron Schmeroffski, daß ich wieder seinen willen were von ihm gereiset, auch der empfangenen 80 thlr. wegen einige Schwierigkeiten machen wolte, so wolten Ihro. Durchl. solches restituiren. Das Eydt, welches von mir vi et metu were extorquiret worden, were bloß geschehen, daß sie mich gerne behalten wolten: in summa Ihr. Durchl. haben mich bis daher durch auftrag allerhand Commissionen wieder nach Dresden zu gehen abgehalten.

I Man vergleiche hierzu den Anfang des Berichtes. 
Kurtz zuvor, da ich von Dresden wil wegreisen, erzehlet mir der junge Glaser alß studiosius(!) juris die Sache, wie es Frenckel angefangen. AlB derselbe von dem Fürst zu Arnstadt ist wieder abgeschicket worden, mich aus Schlesien von vorgedachten Baron abzuhohlen, gehet Frenckel zu Dr. Glasern, eröffnet demselben, daß er eine Person aus Schlesien abhohlen, und wann er wüste, daß er ein praesent bekäme, so wolte er diesen Menschen auf Dresden führen. Doctor Glaser macht Frenckeln adresse an dem Cammer Raht Neimitz. Der Cammer Raht mag solches denen Geheimbten Rähten proponiren. Frenckel bekomt resolution, er könte die Person überbringen, wegen eines praesents müste er so lang in Gedult stehen, bis Ihro Königl. Majestät aus Pohlen wieder zurück kämen.

Nachdem ich nun solches alles erfahren, hielt ich es dem Frenckel vor. Frenckel konte nicht in abrede seyn, baht mich hertzlich, ich solte es ihm dieses mahl verzeihen, er wolte lebenslang dergleichen nicht wieder gegen mir vornehmen, es were nicht recht angefangen. Er hette gemeinet, man würde es mit höfflichkeit angefangen haben.

Endlich habe ich auch durch Dr. Glasers Sohn im Ambte zufragen laßen, ob ich auch noch nöhtig hette, vor dem Ambte zu erscheinen; bekam zur Antwortt, daB ich nicht mehr erscheinen dürfte, weiln ich über die puncta, was Weideman, den Haubtmann anlangete, meine ausrede schon gethan hette, und solte mir auch wegen des Eydes keine bekümmernis machen, weil es pro absolutorio geachtet würde, und were deswegen geschehen, daß ich nicht nach Arnstadt gehen solte, weil sie mich gerne behalten wolten.

Martin Apollo Dantzig. 